\title{
Wildcat World: Simulation programs for teaching basic concepts in psychological science
}

\author{
MICHAEL F. BROWN \\ Villanova University, Villanova, Pennsylvania
}

\begin{abstract}
A series of computer programs is described that allows beginning psychology students to design, conduct, analyze, and interpret virtual (computer-simulated) psychological studies. This technique allows the instructor more control over the outcome of student experiments, increases the scope of experiments that can be done by students, decreases the amount of class time that must be devoted to conducting experiments, and eliminates concerns about student experimenters using human or animal subjects.
\end{abstract}

Two psychology professors teach courses in research methods or experimental psychology. Among the goals of their courses is to provide students with a deep understanding of the purposes, assumptions, advantages, and disadvantages of correlational and experimental research. Professor A does this by having students plan and carry out their own psychological experiments. Although there are clear pedagogical advantages to this heroic, tried-andtrue technique, Professor A is often distressed by the lack of control that she has over the outcome of these experiments. Often student learning is limited by the large number of experiments that "don't work" and therefore illustrate only some of the concepts that they are intended to illustrate. "Having students plan and conduct experiments is a good thing," thinks Professor A, "but it would be nice to have a bit more control over the results of the experiments." Meanwhile, Professor B uses a different set of teaching techniques. He lectures about the principles of variation, covariation, experimental manipulation, and the patterns of inferences underlying various types of psychological research. "My students are exposed to sophisticated knowledge about research principles," muses Professor B, "but they have little or no hands-on experience doing it."

This parable illustrates a fundamental dilemma in the teaching of the basic concepts of behavioral research. Psychological science, like so many things, is probably best learned by doing. But, as teachers, we want to make sure that our students obtain data in these initial experiments that will illustrate the concepts we want them to learn and encourage them to do additional experiments. This paper

\footnotetext{
"Wildcat" refers to the mascot of Villanova University. I thank all the students in Psychology 4050 (Research Methods in Psychology) during the past decade who have inspired and supported the creation and development of Wildcat World. A copy of the Wildcat World programs may be obtained on the internet as www.vill.edu/ mbrown/abstract.htm or by sending a blank diskette to M.F.B. along with your request. These programs are compatible with any personal computer running Windows Version 3.1 or later. Correspondence should be addressed to M. F. Brown, Department of Psychology, Villanova University, Villanova, PA 19085 (e-mail: mbrown@email.vill.edu).
}

describes one attempt to solve this dilemma in a limited domain. The underlying strategy is very simple: the use of computer-based simulated research studies, which allow the instructor to restrict or control the outcome of the studies performed by the students. Thus, although students plan the details of the study (e.g., they decide what the research question is and which variables need to be manipulated in order to answer it), the outcome of the study is restricted within boundaries determined by the instructor. Studies can be carried out very quickly and do not require the use of actual human or animal subjects (an advantage in these days of tight restrictions related to the use of subjects).

\section{THE BASICS OF WILDCAT WORLD}

Early versions of Wildcat World were written using Microsoft BASIC, but the present version was written using Microsoft Visual Basic for Windows Professional Edition, Version 3.0 (Microsoft, 1993). The basic premise of Wildcat World, from the students' perspective, is that it involves the study of human facial features. In each of the three modules, features of cartoon human faces (shape of the eyes, shape of the mouth, size of a laugh as represented by the size of the mouth) are measured as dependent variables. These measures are taken either by judgments made by the students or by "instruments" that are part of the Wildcat World module. In the first module of Wildcat World, two dependent measures are taken by student judgments, judgments are compared to illustrate the concepts involved in interrater reliability, and the two measures are compared to determine whether there is a correlation between them. In the second module, students design an experiment in which one independent variable is manipulated in order to determine its effect on an automated measure of mouth size (laugh size). In the third module, a "complex" experiment is designed in which two independent variables are manipulated. The results obtained in this experiment are constrained in such a way that students obtain an interaction between the effects of the two independent variables. 


\section{WILDCAT WORLD I: MEASUREMENT, INTERRATER RELIABILITY, AND CORRELATION}

Figure 1 shows a sample screen from Wildcat World I. The caricature of the human face serves as the common feature of Wildcat World. The face appears on each "trial" of the "study," with the eyes and mouth animated to slowly open to a terminal size and shape, which varies from trial to trial. The degree and nature of the variation in these measured variables is determined by the instructor/programmer. I have often found it useful to maximize variation by simply using the BASIC RND function to produce an evenly distributed value between $-n$ and $n$, which is then added to the intended mean value of the variable. Positive and negative correlations between the two variables can be produced by restricting the value of one variable depending on the value of the other. However, when students are to apply inferential statistics to their results, values of the two variables are made to vary according to a normal distribution, using the technique of Box and Muller (1958).

Students control the pace of the study in that the program advances to the next "trial" when the student uses the computer mouse to click on the button labeled "next face." Each trial consists simply of exposure to a face, and faces vary from trial to trial in terms of two features: the shape of the eyes and the shape of the mouth. These variables are implemented in Visual Basic as the aspect ratio of the circles that form the facial features. On each trial, a pair (or more) of students independently rates the eye and mouth shape on a 1-100 scale, using the scale anchors shown on the left of the screen. Two activities form the basis of the laboratory exercise I typically use with this mod- ule. First, students develop and obtain a measure of interobserver reliability by comparing the ratings of different student raters. Second, they plot and calculate the degree of correlation between their measure of eye shape and mouth shape. I have usually programmed Wildcat World I so that there is a moderate positive correlation, a moderate negative correlation, or no correlation between these features. A code appears prior to the first trial that specifies which of these three (randomly determined) conditions obtains during that run of the program (if students are instructed to record this code, it allows the instructor to know the [approximate] correlation between the variables for that run of the program). The faces presented on different trials can be described to students as representing different people (to see whether eye shape and mouth shape are correlated across people) or as one person on different occasions (to see if an individual's mouth and eyes covary in shape).

The intent of this program is to provide a mechanism for efficiently demonstrating the concepts of variation, the need for judgment in behavioral measures, the purpose of measures of interobserver agreement, and correlation as a measure of covariation among variables.

\section{WILDCAT WORLD II: EXPERIMENTAL MANIPULATION}

In the second module, Wildcat World is presented to students as representing a research project carried out in a comedy club. The face now represents patrons of the comedy club whose reactions to comedians on stage are measured. The dependent variable is the size of the laugh produced by the comedian in each patron, and this is measured automatically by an instrument identified as a "mouthom-

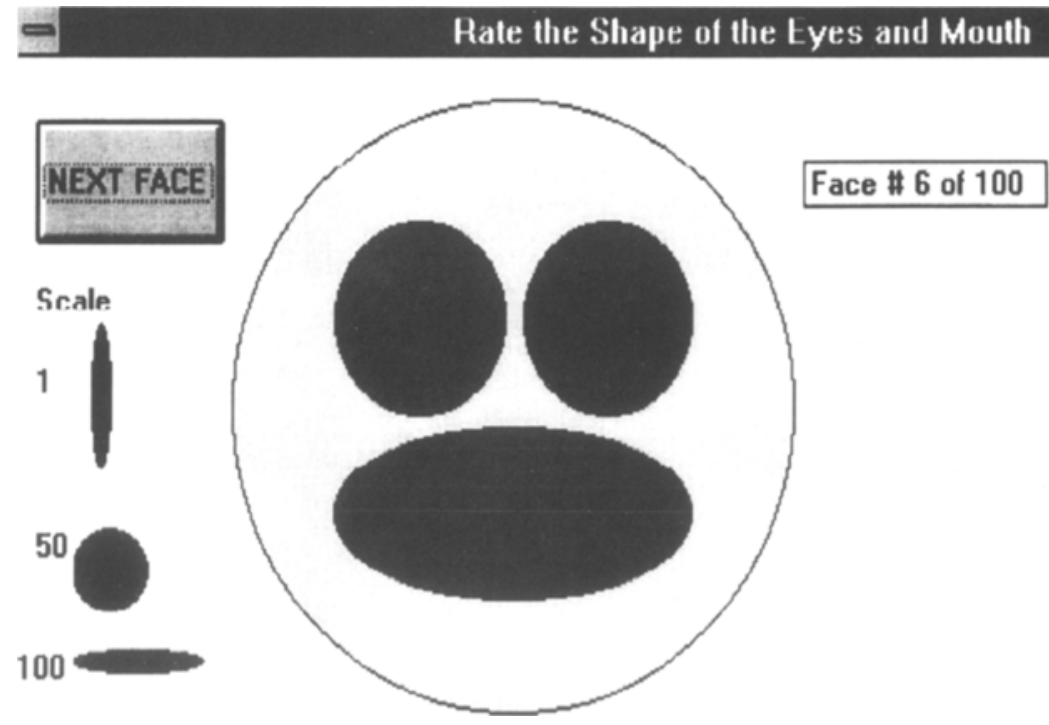

Figure 1. Screen capture of display during a trial of Wildcat World I. Button in upper left corner of display allows advance to next face, using mouse click. Ellipses on left provide anchors for rating scale to be applied to mouth/eye shape. 
eter" or "laughometer." The student/experimenter is given complete control over the identity of the comedian appearing on stage, selected from four possibilities, including a no-comedian control condition. On each trial, the level of the independent variable must be chosen (student experimenters use the mouse to choose a level of comedian from the menu presented in the bottom left area of the screen; see Figure 2). The trial is then activated by clicking on the "next trial" button in the top left area of the screen. The resulting value of the dependent variable (mouth/laugh size) is measured by the "mouthometer" in the bottom right area of the screen. I have typically programmed this value to vary moderately around mean values that differ among levels of comedian, with some comedians producing mean values substantially greater than that produced by the control condition and other comedians producing mean values that do not differ from the mean produced by the control condition.

Thus, the student experimenters decide what experimental question their experiment is to address (e.g., comparison of the effect of two different comedians, determination of whether a particular comedian is at all funny). This module can be presented as representing manipulation of the independent variable either between subjects or within subjects. Students have to determine the order in which conditions are to be presented, considering issues such as order effects (e.g., time of day, audience fatigue). Although I have not typically programmed an order effect into the functions producing the data, this can be easily done. In either case, the possibility of order effects is included in the discussion of the project when it is presented as involving within-subjects manipulation. Thus, concepts such as trial block randomization can be included in the context of designing this Wildcat World experiment.

\section{WILDCAT WORLD III: COMPLEX EXPERIMENTS AND INTERACTIONS}

The third module of Wildcat World expands on the concepts presented in the second module. Again, the module is presented as representing an experiment in which the experimenter has complete control over the identity of the comedian appearing on stage in a comedy club. In addition, the student experimenter has complete control over the number of companions with whom the subject is sitting. As shown in Figure 3, this second independent variable is represented on the screen as a schematic of a round table, with a number of squares representing occupied chairs at the table. The subject is sitting at a table alone, sitting with one companion (two persons at the table), or sitting with five companions (six at the table). Thus, on each trial of the virtual experiment, the student experimenter selects a level for each of the two independent variables (identity of the comedian on stage and number of people at the subject's table). After invoking the trial (using the "next trial" button), the size of the mouth/laugh produced in the subject is given by the mouthometer.

Typically, I have programmed this module so that the effects of independent variables tend to produce an interaction. This is accomplished by programming the value of the dependent variable to vary around a mean that is a multiplicative function of the values of the two independent variables. Students have typically been encouraged to design $2 \times 2$ experiments, at least initially. Thus, they usually obtain a marked interaction between the effects of social condition and comedian identity. Discussion of the meaning and possible interpretation of the interactions obtained provides a framework for understanding the importance of interaction in designing and interpreting psy-

Live Comedy Tonight!

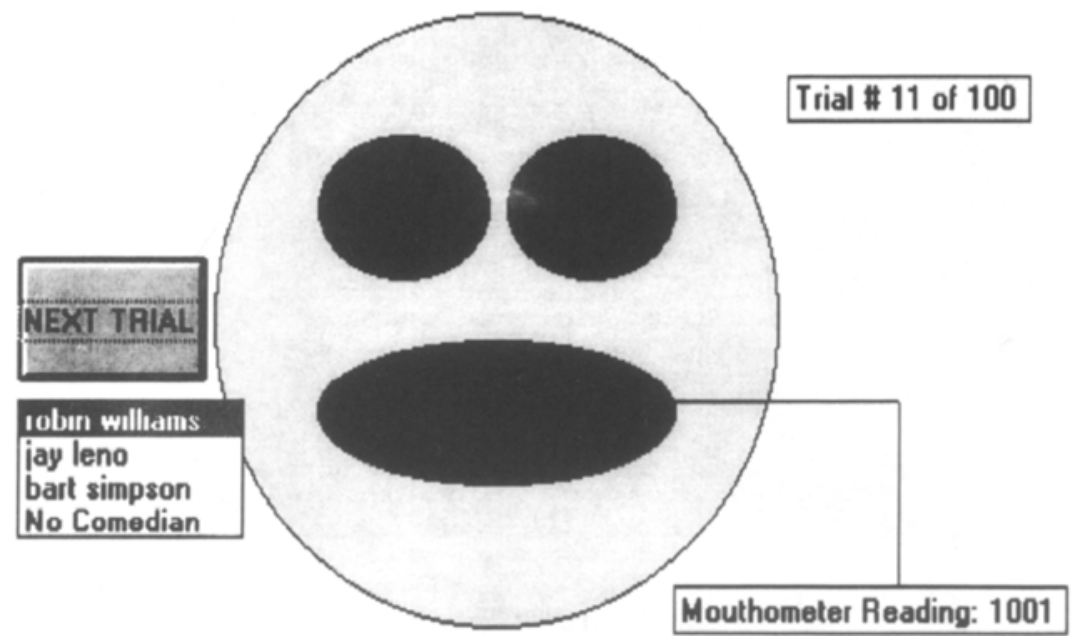

Figure 2. Screen capture of display during a trial of Wildcat World II. Button on left side of display allows advance to next trial, using mouse click. Menu on lower left side allows choice of the level of the independent variable (comedian), using mouse click. The measure provided in the box in the lower right corner of the screen provides resulting value of dependent variable (mouth/laugh size). 


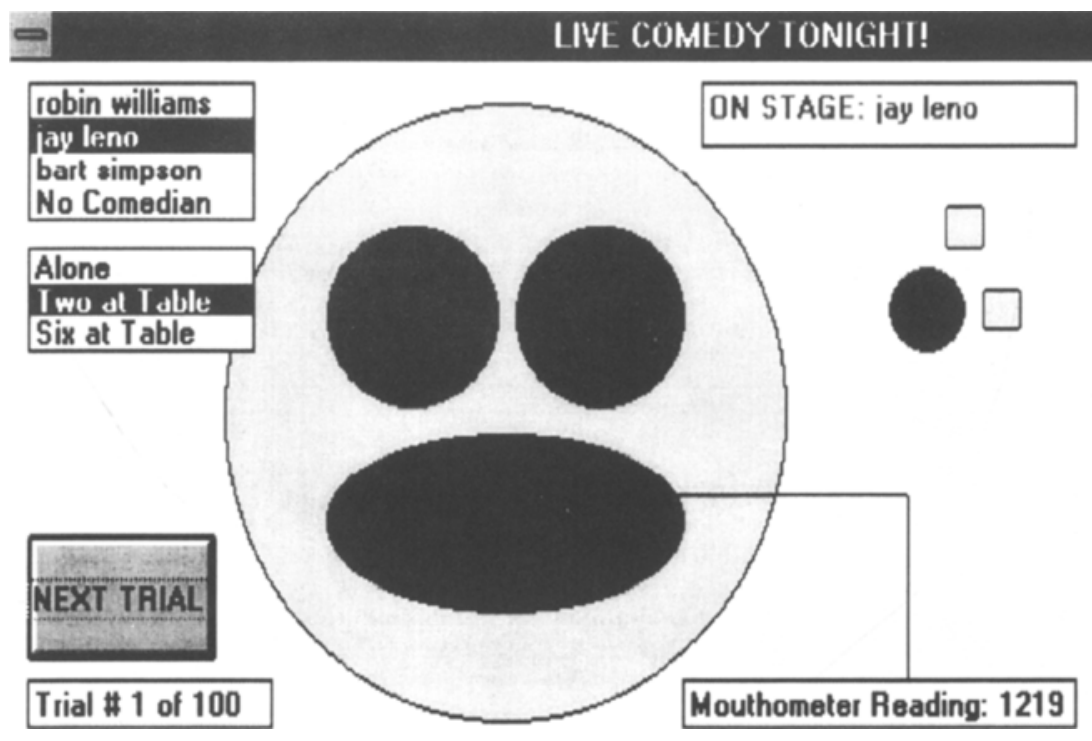

Figure 3. Screen capture of display during a trial of Wildcat World III. Button in lower left corner of display allows advance to next trial, using mouse click. Menus in upper left corner allow choice of the levels of the two independent variables (comedian and number of companions), using mouse clicks. The values of these independent variables are displayed in the upper right corner of the display. The measure provided in box in lower right corner of the screen provides resulting value of dependent variable (mouth/laugh size).

chological experiments. For example, students discover that the form of the interaction between the effects of the two variables can be specified only by examining a larger number of experimental conditions.

\section{COMMENTARY ON WILDCAT WORLD}

Computer simulations used in the teaching of psychology have been described for a number of specialized content areas, such as operant conditioning (Graf, 1995; Graham, Alloway, \& Krames, 1994; Shimoff \& Catania, 1995). Computer simulations have also been described that use stochastically determined events to illustrate statistical principles (see, e.g., Goldstein \& Strube, 1995; Strube \& Goldstein, 1995). There is some empirical evidence that such programs are effective in helping to communicate such concepts to students (Weir, McManus, \& Kiely, 1991).

Wildcat World is based on using these techniques, in a context that avoids any particular area of psychological content, to highlight the basic concepts underlying psychological science. It is clear to students that the specific variables and measures involved in Wildcat World are irrelevant and that the points to be learned from the exercises have to do with general methodological principles. Thus, the often difficult-to-understand concepts of variation, covariation, experimental control, interaction, and so on are emphasized rather than any specific content area of psychology. Experiments can be carried out very quickly, and the results of the experiments can be controlled or restricted by the instructor. Finally, students can design, conduct, and interpret psychological studies without using human or animal subjects.
Reaction to Wildcat World by Villanova University students has been positive. A recent survey of students who had used Wildcat World during the immediately preceding semester asked them to rate each of the three modules in terms of "the extent to which it effectively illustrated..." "the use of ratings, measures of interrater reliability, and the logic of correlational research" (Wildcat World I); "the basic features of a simple experiment" (Wildcat World II); or "complex experiments (i.e., experiments with more than one independent variable" (Wildcat World III). Responses were provided anonymously on a 5-point Likerttype scale $(1=$ poorly, $3=$ moderately, and $5=$ very well $)$. The mean responses $(n=13)^{1}$ were $4.1,4.2$, and 3.6 , respectively.

These simulations of basic research methodologies are a valuable technique for providing hands-on research experience that avoids the disadvantages and complications of having students use live subjects during their initial experiences as experimenters. Advantages of this approach include the ability of the instructor to control the outcome of student projects, the relatively small amount of time required for data collection, and the explicit emphasis on the methodological issues rather than particular content areas represented by experimental paradigms that might otherwise be used. These advantages make Wildcat World, or software based on the same principles as Wildcat World, a valuable tool for a research methods or experimental psychology course.

\section{REFERENCES}

Box, G. E. P., \& MUlLer, M. E. (1958). A note on the generation of random normal deviates. Annals of Mathematical Statistics, 29, 610-611. 
Goldstein, M. D., \& Strube, M. J. (1995). Understanding correlations: Two computer exercises. Teaching of Psychology, 22, 205-206.

Graf, S. A. (1995). Three nice labs, no real rats: A review of three operant laboratory simulations. Behavior Analyst, 18, 301-306.

Graham, J., Alloway, T., \& Krames, L. (1994). Sniffy, the virtual rat: Simulated operant conditioning. Behavior Research Methods, Instruments, \& Computers, 26, 134-141.

Microsoft Visual BASIC 3.o [Computer software] (1993). Redmond, WA: Microsoft.

Shimoff, E., \& Catania, A. C. (1995). Using computers to teach behavior analysis. Behavior Analyst, 18, 307-316.

Strube, M. J., \& Goldstein, M. D. (1995). A computer program that demonstrates the difference between main effects and interactions. Teaching of Psychology, 22, 207-208.
Weir, C. G., McManus, I. C., \& Kiely, B. (1991). Evaluation of that teaching of statistical concepts by interactive experience with Monte Carlo simulations. British Journal of Educational Psychology, 61, 240-247.

\section{NOTE}

1. Surveys were provided to students in a class of 25 who had taken Research Methods in Psychology the previous semester. Students were asked to complete the survey following that class and return it to a faculty mailbox. Thus, the return rate was $52 \%$.

(Manuscript received August 15, 1997; revision accepted for publication May 28, 1998.) 